\title{
Joule heating in nanowires
}

\author{
Hans Fangohr, ${ }^{1, *}$ Dmitri S. Chernyshenko, ${ }^{1}$ Matteo Franchin, ${ }^{1}$ Thomas Fischbacher, ${ }^{1}$ and Guido Meier ${ }^{2}$ \\ ${ }^{1}$ School of Engineering Sciences, University of Southampton, SO17 1BJ, Southampton, United Kingdom \\ ${ }^{2}$ Institut für Angewandte Physik und Zentrum für Mikrostrukturforschung, \\ Universität Hamburg, Jungiusstrasse 11, 20355 Hamburg, Germany
}

\begin{abstract}
We study the effect of Joule heating from electric currents flowing through ferromagnetic nanowires on the temperature of the nanowires and on the temperature of the substrate on which the nanowires are grown. The spatial current density distribution, the associated heat generation, and diffusion of heat is simulated within the nanowire and the substrate. We study several different nanowire and constriction geometries as well as different substrates: (thin) silicon nitride membranes, (thick) silicon wafers, and (thick) diamond wafers. The spatially resolved increase in temperature as a function of time is computed.

For effectively three-dimensional substrates (where the substrate thickness greatly exceeds the nanowire length), we identify three different regimes of heat propagation through the substrate: regime (i), where the nanowire temperature increases approximately logarithmically as a function of time. In this regime, the nanowire temperature is well-described analytically by You et al. [APL89, 222513 (2006)]. We provide an analytical expression for the time $t_{\mathrm{c}}$ that marks the upper applicability limit of the You model. After $t_{\mathrm{c}}$, the heat flow enters regime (ii), where the nanowire temperature stays constant while a hemispherical heat front carries the heat away from the wire and into the substrate. As the heat front reaches the boundary of the substrate, regime (iii) is entered where the nanowire and substrate temperature start to increase rapidly.

For effectively two-dimensional substrates (where the nanowire length greatly exceeds the substrate thickness), there is only one regime in which the temperature increases logarithmically with time for large times, before the heat front reaches the substrate boundary. We provide an analytical expression, valid for all pulse durations, that allows one to accurately compute this temperature increase in the nanowire on thin substrates.
\end{abstract}

\section{INTRODUCTION}

Recently, there has been much interest both in fundamental studies of spin torque transfer ${ }^{115}$ and in efforts to realize devices such as the race-track memory exploiting the spin torque transfer ${ }^{6 / 7}$ In either case, at the present very large current densities have to be used to move domain walls and, more generally, to modify the ferromagnetic patterns. Associated with these large current densities is a substantial amount of Joule heating that increases the temperature of the sample or device. It is a crucial question to understand how strongly the temperature increases as this can affect the observed physics considerably, e.g. Refs. 8 10, and may even lead to a temporary breakdown of ferromagnetism if the Curie temperature is exceeded. The depinning of a domain wall could be due to a strong spin-current torque transfer, or as a result of the extreme heating of the material due to reduced magnetic pinning at elevated temperatures, or due to the intermittent suppression of ferromagnetism.

You et al $!^{11}$ have derived an analytical expression that allows to compute the increase of temperature for a nanowire (extending to plus and minus infinity in ydirection) with height $h$ (in z-direction) and width $w$ (in $\mathrm{x}$-direction). The nanowire is attached to a semi-infinite substrate (which fills all space for $z \leq 0$ ). The heating is due to an explicit term $S(x, t)$ which can vary across the width of the wire and as a function of time.
Meier et al ${ }^{[4}$ use energy considerations to estimate the total amount of energy deposited into the nanowire and substrate system to show that - for their particular parameters - the heating and associated temperature increase stays below the Curie temperature.

In this work, we use a numerical multi-physics simulation approach which allows to determine the temperature distribution $T(\mathbf{r}, t)$ for all times $t$ and positions $\mathbf{r}$. Starting from a given geometry and an applied voltage (or current), we compute the resulting current density, the associated heat generation, and the temperature distribution. While such a numerical approach provides less insight than an analytical approximation, it allows us to exactly determine the temperature distribution for nanowires of finite length, nanowires with constrictions and thin substrates for which the assumption of an infinite thickness is inappropriate. Thus it evinces the limits of applicability of analytical approximations.

While the simulation and analytical techniques used and developed in this work are not limited to ferromagnetic nanowires on substrates, we have chosen materials, geometries and current densities that are typical for spintorque driven domain wall motion studies in ferromagnetic nanowires in order to illuminate the role of Joule heating in this active research area 10

Section II introduces the method underlying the work. Section III reports results from a number of case studies. Starting from the heating of a nanowire of infinite 
length without constrictions (Sec. III A), we introduce a symmetric constriction in a finite-length wire where a cuboidal part of material has been removed (Sec. IIIB) to demonstrate the additional heating that results from an increased current density in close proximity to the constriction and in the constriction (as in Ref. 12). Section IIC studies a nanowire with a notch-like constriction (triangular shape removed from the wire on one side only) that is placed on a silicon nitride substrate of $100 \mathrm{~nm}$ thickness (as in Ref. 13 15). The same system is studied in Sec. IIID where the silicon nitride membrane is replaced with a silicon wafer with a thickness of the order of $500 \mu \mathrm{m}$. A zigzag wire on the same silicon wafer, as experimentally investigated in Ref. 3, is studied in Sec. IIIE, We simulate a straight nanowire without constrictions placed on a diamond substrate as in Ref. 16 in Sec. IIIF. In Sec. IV A we investigate and discuss the applicability of the analytical temperature calculation model of Ref. 11, and deduce an analytical model valid for quasi two-dimensional systems such as membrane substrates in Sec. IVB. We briefly discuss freestanding and perpendicular nanowires in Sec. V], before we close with a summary in Sec. VI.

\section{METHOD}

A current density $\mathbf{j}(\mathbf{r}, t)$ can be related to the change of temperature $T(\mathbf{r}, t)$ of a material as a function of position $\mathbf{r}$ and time $t$ using

$$
\frac{\partial T}{\partial t}=\frac{k}{\rho C} \nabla^{2} T+\frac{Q}{\rho C}=\frac{1}{\rho C}\left(k \nabla^{2} T+Q\right)
$$

with $k$ the thermal conductivity $(\mathrm{W} /(\mathrm{Km})), \rho$ the density $\left(\mathrm{kg} / \mathrm{m}^{3}\right), C$ the specific heat capacity $(\mathrm{J} /(\mathrm{kg} \mathrm{K}))$, $\nabla^{2}=\frac{\partial^{2}}{\partial x^{2}}+\frac{\partial^{2}}{\partial y^{2}}+\frac{\partial^{2}}{\partial z^{2}}$ the Laplace operator, $T$ the temperature $(\mathrm{K})$, and $Q$ a heating term $\left(\mathrm{W} / \mathrm{m}^{3}\right)$. The Joule heating of a current density $\mathbf{j}$ in an electrical field $\mathbf{E}$ is given by

$$
Q=\mathbf{j} \cdot \mathbf{E}=\frac{1}{\sigma} \mathbf{j}^{2}
$$

where $\sigma$ is the electrical conductivity $(\mathrm{S} / \mathrm{m}=1 /(\Omega \mathrm{m}))$ and we have used $\mathbf{j}=\sigma \mathbf{E}$.

Equation (1) becomes trivial to solve if we assume a uniform current density in a slab of one material with constant density, constant thermal conductivity, and constant specific heat capacity (see Sec. III A). In general, for samples with geometrical features or spatially inhomogeneous material parameters, the problem becomes quite complex and can often only be solved using numerical methods. For the work presented here we have used the simulation software suites ANSYS 12.0ㄹ. Comsol multi-physics, $\frac{18}{18}$ and the Nsim multiphysics simulation library $\sqrt{19}$ that underpins the $\mathrm{Nmag}^{20}$ micromagnetic simulation package. All three tools were used for case study 2 (Sec. IIIB) and produce identical results for a given desired accuracy within their error tolerance settings. The majority of the other case studies was simulated using ANSYS. We have taken material parameters (see Tab. II) appropriate for room temperature, and have treated each material parameter as a constant for each simulation, i.e. here a temperature dependence of the material parameters is not taken into account.

\section{SIMULATION RESULTS}

Case studies 1 and 2 (Sec. III A and III B investigate a nanowire without a substrate. Case study 3 (Sec. III C studies a nanowire on $(2 \mathrm{~d})$ silicon nitride substrate membrane. Case studies 4 and 5 (Sec. IIID and Sec. III E) investigate nanowires on (3d) silicon wafer substrates, and case study 6 (Sec. IIIF) reports from a nanowire on a (3d) diamond substrate.

We refer to the substrate as two-dimensional where the wire length is much greater than the substrate thickness (but still carry out numerical calculations by discretising space finely in all three dimensions). We call the substrate three-dimensional if the substrate thickness is much greater than the wire length.

\section{A. Case study 1: Uniform current density}

Initially, we study the extreme case of no cooling of the ferromagnetic conductor: neither through heat transfer to the surrounding air, nor to the substrate and the contacts. This allows to estimate an upper limit of the heating rate and the consequent change in temperature over time.

Assuming a uniform current density $\mathbf{j}$, uniform conductivity $\sigma$ and initially uniform temperature distribution $T$, equation (1) simplifies to

$$
\frac{\mathrm{d} T}{\mathrm{~d} t}=\frac{j^{2}}{\rho C \sigma}
$$

All the parameters on the right hand side are constant, and thus the temperature $T$ will change at a constant rate of $j^{2} /(\rho C \sigma)$. As only the ferromagnetic conducting nanowire can store the heat from the Joule heating, the temperature has to increase proportionally to the heating term $Q$ which is proportional to $j^{2}$.

Using material parameters for Permalloy $(C=$ $430 \mathrm{~J} /(\mathrm{kg} \mathrm{K}), \rho=8700 \mathrm{~kg} / \mathrm{m}^{3}, \sigma=1 /\left(25 \cdot 10^{-8} \Omega \mathrm{m}\right)=$ $\left.4 \cdot 10^{6}(\Omega \mathrm{m})^{-1}, j=10^{12} \mathrm{~A} / \mathrm{m}^{2}\right)$, we obtain a change of temperature with time

$$
\frac{\mathrm{d} T}{\mathrm{~d} t}=\frac{j^{2}}{\rho C \sigma}=6.683 \cdot 10^{10} \mathrm{~K} / \mathrm{s}=66.83 \mathrm{~K} / \mathrm{ns} .
$$

The parameters for this case study 1 and all other case are summarised in Tab. I. For the permalloy wire, we have chosen parameters for case studies 1 to 4 to match the experimental work in Ref. 13 15. Where possible, 


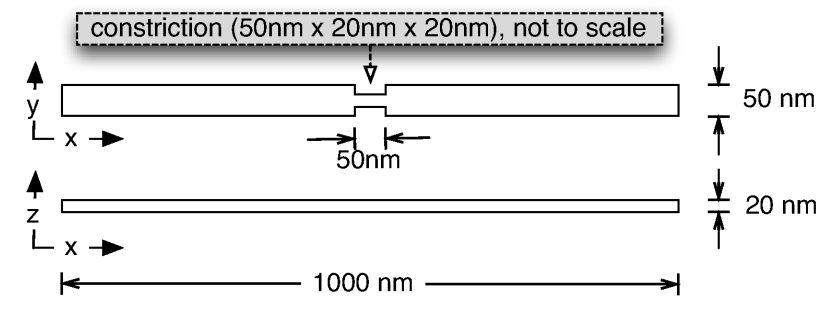

FIG. 1. Sample geometry for case study 2: slab with constriction (Sec. III B).

parameters measured as part of the experiments have been used and have been complemented with literature values (see Tab. I for details).

The immediate conclusion from this is that the temperature of the sample cannot increase by more than $66.83 \mathrm{~K}$ per nanosecond if the current density of $10^{12} \mathrm{~A} / \mathrm{m}^{2}$ is not exceeded and if the current density is uniform within the whole sample for the chosen material parameters.

A current pulse over 15 nanoseconds has the potential to push the temperature up by just over 1000 degrees Kelvin, and thus potentially beyond the Curie temperature.

The substrate on which the ferromagnetic conductor has been grown will absorb a significant fraction of the heat generated in the conductor, and thus reduce the effective temperature of the magnetic material. The contacts play a similar role. On the other hand, any constrictions will result in a locally increased current density, which - through the $j^{2}$ term in $Q$ in Eq. (2) and (1) - results in significantly increased local heating. We study the balance of these additional heating and cooling terms in the following sections in detail.

\section{B. Case study 2: Constrictions}

The effect of a constriction will vary strongly depending on the given geometry. The resulting current density distribution and spatial and temporal temperature distributions are non-trivial.

Fig. 11 shows the geometry used for this study (as in Refs. 12, 32, and 33): a bar with dimensions $L_{\mathrm{x}}=$ $1000 \mathrm{~nm}, L_{\mathrm{y}}=50 \mathrm{~nm}$, and $L_{\mathrm{z}}=20 \mathrm{~nm}$. The origin is located in the center of the slab, i.e. the two opposite corners of the geometry are at $[-500,-25,-10] \mathrm{nm}$ and $[500,25,10] \mathrm{nm}$. The constriction is placed at the center of the bar, and reduces the dimensions to $L_{\mathrm{y}}^{\text {constrict }}=$ $20 \mathrm{~nm}$ over a length of $L_{\mathrm{x}}^{\text {constrict }}=50 \mathrm{~nm}$. In comparison to case study 1 (Sec. III A), the current distribution is non-uniform in this geometry and therefore the local Joule heating and the resulting temperature field will be non-uniform. We thus need the thermal conductivity for $\mathrm{Ni}_{80} \mathrm{Fe}_{20}$ permalloy $k=46.4 \mathrm{~W} /(\mathrm{K} \cdot \mathrm{m})$ for these calculations because the $\nabla^{2} T$ term in equation $(1)$ is non-zero.

Fig. 2 shows a temperature profile $\Delta T(x)$ through the constricted shape after application of a current density

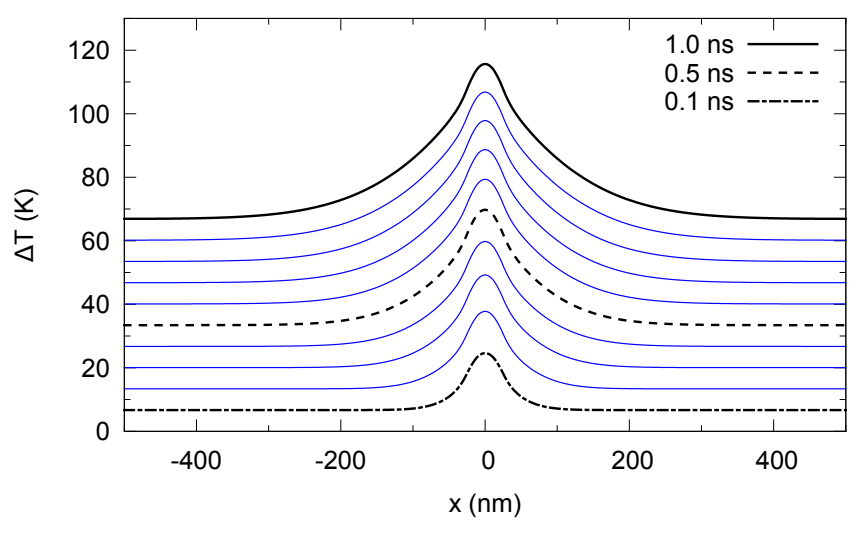

FIG. 2. Temperature profile $\Delta T(x)$ in the constricted geometry shown in Fig. 1 at positions $[\mathrm{x}, 0,0]$ for $t=1 \mathrm{~ns}$ and a current density of $10^{12} \mathrm{~A} / \mathrm{m}^{2}$ in the unconstricted ends of the slab.

of $10^{12} \mathrm{~A} / \mathrm{m}^{2}$ at ten different times $t$ as a function of position $x$. We use the notation $\Delta T(x)$ instead of $T(x)$ to indicate that this is the change of the temperature $T$ relative to the initial temperature, for which we assume room temperature $(\approx 300 \mathrm{~K})$. The top thick black line shows the temperature distribution after $1 \mathrm{~ns}$, the other 9 lines show earlier moments in time in successive time steps of $0.1 \mathrm{~ns}$.

The peak around $x=0$ is due to the constriction: the reduced cross section (in the $\mathrm{y}-\mathrm{z}$ plane) results in an increased current density in the constriction. The Joule heating term 2 - which scales proportional to $j^{2}$ is increased accordingly in the constriction. During the $1 \mathrm{~ns}$, diffusion of heat takes place and results in the increase of temperature outside the constriction, and simultaneously a reduction of the speed of increase of the temperature in the constriction. The importance of heat diffusion can be seen by the width of the peak around $x=0$ in Fig. 2 .

The increase of temperature $\Delta T$ after $t=1 \mathrm{~ns}$ at the end of the nanowire (i.e. $x=500 \mathrm{~nm}$ and $x=-500 \mathrm{~nm}$ ) is $66.90 \mathrm{~K}$. This is about $0.07 \mathrm{~K}$ higher than in the previous case study in Sec. III A where a nanowire without a constriction was studied and the corresponding value of temperature increase is $66.83 \mathrm{~K}$. This difference of $0.07 \mathrm{~K}$ after $1 \mathrm{~ns}$ at the end of the wire originates from the extra heating in the constriction around $x=0$ and diffusion of this heat through the wire.

We can also estimate the maximum possible temperature increase in the constriction by using equation (4) under the assumption that there was no diffusion of heat (i.e. $k=0$ ), and that we obtain a current density of $j=2.5 \cdot 10^{12} \mathrm{~A} / \mathrm{m}^{2}$ in the constriction (due to the reduced cross section from $L_{\mathrm{z}} L_{\mathrm{y}}=1000 \mathrm{~nm}^{2}$ to $L_{\mathrm{z}} L_{\mathrm{y}}^{\text {constrict }}=400 \mathrm{~nm}^{2}$ ). The resulting rate for temperature increase in the constriction is here $417 \mathrm{~K} / \mathrm{ns}$, and thus much higher than the maximum temperature of $115.6 \mathrm{~K}$ that is reached after $1 \mathrm{~ns}$ when taking into account the diffusion of heat from the constriction into the 


\begin{tabular}{|c|c|c|c|c|}
\hline Case Study & Parameter & Value & Unit & Reference \\
\hline $1-4$ & Py $\sigma^{-1}$ & 25 & $\mu \Omega \mathrm{cm}$ & measured $^{21}$ in experiment ${ }^{\frac{14 \mid 15}{15}} R=280 \Omega$ (case study 3 ) \\
\hline 5 & Py $\sigma^{-1}$ & 42 & $\mu \Omega \mathrm{cm}$ & measured in experiment ${ }^{3}, R=5000 \Omega$ \\
\hline 6 & Py $\sigma^{-1}$ & 39 & $\mu \Omega \mathrm{cm}$ & measured in experiment ${ }^{[16}, R=675 \Omega$ \\
\hline $1-6$ & Py $C$ & 0.43 & $\mathrm{~J} /(\mathrm{g} \mathrm{K})$ & Ref. 22 p. $252,6.00 \mathrm{cal} /(\mathrm{mol} \mathrm{K})$ \\
\hline $1-6$ & Py $k$ & 46.4 & $\mathrm{~W} /(\mathrm{K} \mathrm{m})$ & Ref. 23 p. $1140, T=300 \mathrm{~K}$ \\
\hline $1-6$ & Py $\rho$ & 8.7 & $\mathrm{~g} / \mathrm{cm}^{3}$ & Ref. 24 Tab. I, lattice constant $3.54 \AA$ \\
\hline 3 & $\mathrm{Si}_{3} \mathrm{~N}_{4} C$ & 0.7 & $\mathrm{~J} /(\mathrm{g} \mathrm{K})$ & Ref. 25 \\
\hline 3 & $\mathrm{Si}_{3} \mathrm{~N}_{4} k$ & 3.2 & $\mathrm{~W} /(\mathrm{K} \mathrm{m})$ & Ref. 25 \\
\hline 3 & $\mathrm{Si}_{3} \mathrm{~N}_{4} \rho$ & 3 & $\mathrm{~g} / \mathrm{cm}^{3}$ & Ref. 25 \\
\hline 4,5 & Si $C$ & 0.714 & $\mathrm{~J} /(\mathrm{g} \mathrm{K})$ & Ref. 26, $C_{\mathrm{p}}=20.05 \mathrm{~J} /(\mathrm{mol} \mathrm{K})$ \\
\hline 4,5 & Si $k$ & 148 & $\mathrm{~W} /(\mathrm{K} \mathrm{m})$ & Ref. 27 p. I-588 \\
\hline 4,5 & Si $\rho$ & 2.33 & $\mathrm{~g} / \mathrm{cm}^{3}$ & Ref. 28, Tab. III \\
\hline 6 & Diamond $C$ & 0.53 & $\mathrm{~J} /(\mathrm{g} \mathrm{K})$ & Ref. $29, C_{\mathrm{p}}=6.37 \mathrm{~J} /(\mathrm{mol} \mathrm{K})$ \\
\hline 6 & Diamond $k$ & 1400 & $\mathrm{~W} /(\mathrm{K} \mathrm{m})$ & Ref. 30, Fig. 3, Type Ib \\
\hline 6 & Diamond $\rho$ & 3.51 & $\mathrm{~g} / \mathrm{cm}^{3}$ & Ref. 31 \\
\hline
\end{tabular}

TABLE I. Material parameters used in the simulations ( $\sigma$ electric conductivity, $\sigma^{-1}$ electric resistivity, $C$ specific heat capacity, $k$ thermal conductivity, $\rho$ density).

unconstricted parts of the nanowire. This comparison shows the drastic influence of diffusion of heat on the temperatures in the nanowire.

\section{Case study 3: Nanowire with a notch on a silicon nitride substrate membrane}

In this section we investigate a more realistic system following the work by Im et al ${ }^{13}$ for which we take into account the heat dissipation through the substrate. Im et al. study critical external fields for domain wall pinning from constrictions, and subsequent works ${ }^{1415}$ study spintorque driven domain wall motion for this geometry.

Here, we choose the geometry where the domain wall de-pinning field was most clearly defined: a permalloy nanowire with dimensions $L_{\mathrm{x}}=5000 \mathrm{~nm}, L_{\mathrm{y}}=150 \mathrm{~nm}$, $L_{\mathrm{z}}=30 \mathrm{~nm}$ (see Fig. 1(b) in Ref. 13 and lower left subplot of Fig. 3 therein).

The resistivity of a permalloy thin film strongly depends on its thickness; in this simulation we scale the permalloy resistivity $\sigma$ so that the total resistance of the device matches the resistance value $\approx 280 \Omega$ reported 21 for the experiments. ${ }^{14} 15$ This leads to a resistivity $\sigma^{-1}=$ $25 \mu \Omega \mathrm{cm}$, in line with published data on permalloy resistivity (Fig. 1(b) in Ref. 34, $t=30 \mathrm{~nm}$ ). We use the same value for $\sigma$ in case study 1 (Sec. III A), case study 2 (Sec. III B) and case study 4 (Sec. III D) to allow better comparison between the results.

This nanowire is lithographically defined centrally on a silicon nitride membrane that is $100 \mathrm{~nm}$ thick (as in Ref. 13). The membrane used in the experiment ${ }^{13}$ was purchased from Silson Ltd 55 According to the manufacturer the silicon nitride membrane was grown using low pressure chemical vapour deposition (LPCVD).
The thermal properties of LPCVD grown silicon nitride films depend on the details of the growth process as can be seen in the range of parameters being cited in the literature. 25136 We assume values of $k=3.2 \mathrm{~W} /(\mathrm{m} \mathrm{K})$, $C=0.7 \mathrm{~J} /(\mathrm{g} \mathrm{K})$, and $\rho=3 \mathrm{~g} / \mathrm{cm}^{3}$ as in Ref. 25.

For the modelling of the membrane we use the shape of a disk with $0.5 \mathrm{~mm}$ radius and $100 \mathrm{~nm}$ thickness. Preparation of nanostructures on membranes is required for experiments using synchrotron light, that has to transmit through the sample. Such experiments give access to simultaneous time- and space resolution on the nanometer and the sub-nanosecond scale. ${ }^{113}$ Perfect thermal contact between the wire and the silicon nitride substrate disk is assumed. The center of the wire contains a $45 \mathrm{~nm}$ wide triangular notch on one side, and the geometry is sketched in Fig. 3(b) A current density of $10^{12} \mathrm{~A} / \mathrm{m}^{2}$ is applied at the ends of the wire over a time of $20 \mathrm{~ns}$, similar to recent experiments such as in Refs. 14, 15, and 40.

Figure 3(a) shows an overview of the geometry and the computed temperature distribution after 20 ns. The $30 \%$ notch is just about visible on the right hand side of the nanowire halfway between the ends of the wire. The cutplane shown in the right hand side of Fig. 3(a) as an inset shows the temperature distribution in the nanowire in the $y-z$ plane in the center of the constriction (as indicated by a white line and semi-transparent plane in the main plot). Figure $3(\mathrm{~b})$ shows the notch geometry in more detail (not to scale).

Fig. 3(c) shows the temperature profile $\Delta T(x)$ after $20 \mathrm{~ns}$ taken along a line at the top of the nanowire (the same data is encoded in the colours of Fig. 3(a) although more difficult to read quantitatively). We see that most of the wire is at an increased temperature around $270 \mathrm{~K}$. The maximum temperature is found at the constriction: 


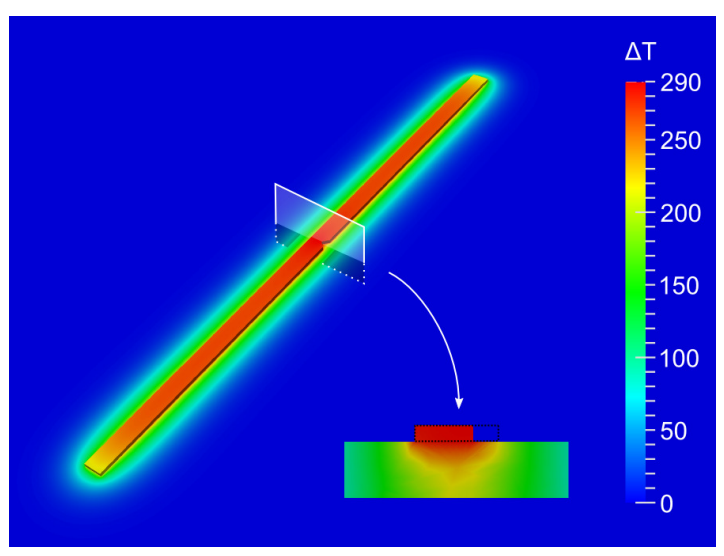

(a)

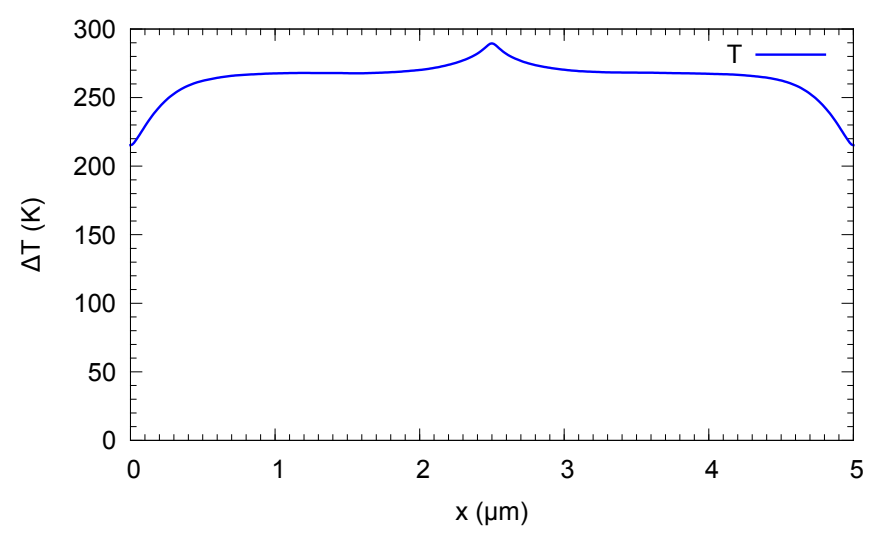

(c)

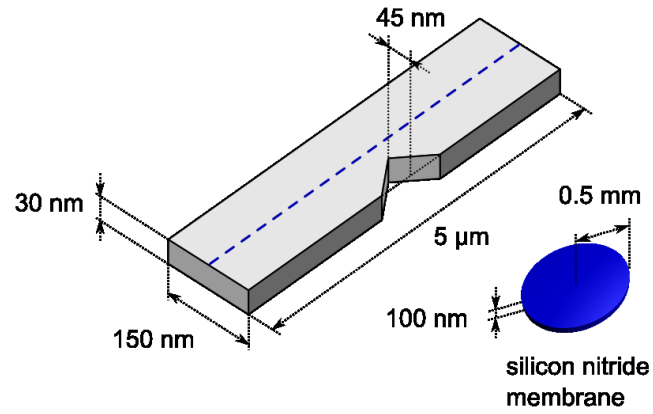

(b)

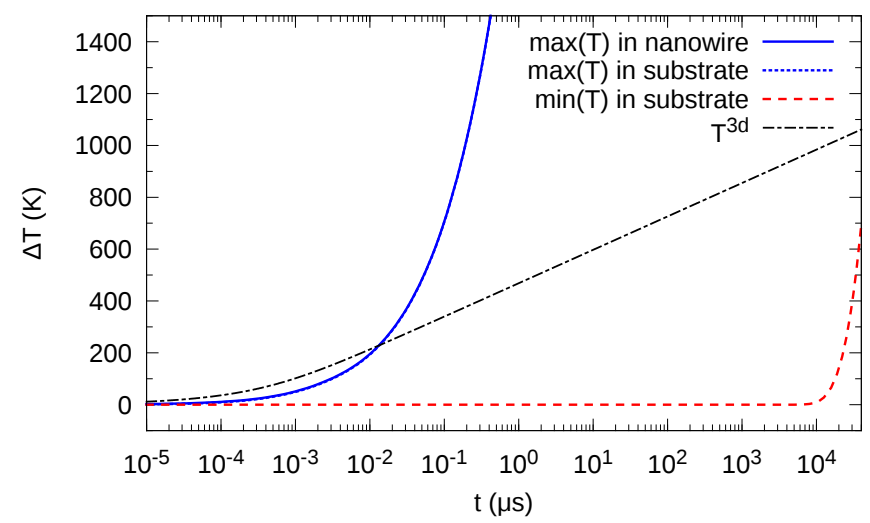

(d)

FIG. 3. Joule heating in a permalloy nanowire with a notch on a silicon nitride membrane ${ }^{13}$ as discussed in Sec. III C The membrane is modelled as a disk with height $100 \mathrm{~nm}$ and radius $0.5 \mathrm{~mm}$. The current density is $j=10^{12} \mathrm{~A} / \mathrm{m}^{2}$; for material parameters see Tab. I. (a) temperature distribution $\Delta T(\mathbf{r})$ in the nanowire and substrate after $t=20 \mathrm{~ns}$, (b) geometry of the model (not to scale) and the plotting path through the nanowire (dashed line) used in Fig. 3(c) (c) temperature profile $\Delta T(x)$ after $t=20 \mathrm{~ns}$ along the length of the wire (following the plotting path shown in Fig. 3(b)) with a silicon nitride membrane substrate, (d) maximum (dotted line) and minimum (dashed line) temperature in the silicon nitride membrane and maximum temperature (solid line) in the wire as a function of current pulse length. The solid and dotted line coincide at this scale. The dash-dotted line $T^{3 \mathrm{~d}}$ is the prediction of the analytical You model discussed in Sec. IV A See also Sec. IV B and Fig. 7 for further discussion.

as in Sec. IIIB the current density is increased here due to the reduced cross section in the y-z plane.

In contrast to the previous example (Sec. IIIB and Fig. 2), the temperature peak at the constriction is less pronounced, as a smaller amount of material is absent and consequently the current density and the associated increase in Joule heating is smaller.

The maximum temperature increase reaches $290 \mathrm{~K}$. The zero level in the simulation corresponds to the temperature at which the experiment is started: if the experiment is carried out at $300 \mathrm{~K}$ we expect the maximum temperature to be $590 \mathrm{~K}$ after $20 \mathrm{~ns}$, which is below the Curie temperature $(\approx 840 \mathrm{~K})$ for Permalloy ${ }^{41}$

From Figs. 3(a) and 3(c) we can see that the temperature at the ends of the wire is lower than near the middle: for $x \lesssim 1 \mu \mathrm{m}$ and $x \gtrsim 4 \mu \mathrm{m}$ temperature decreases towards $\approx 215 K$ at the ends of the wire. This is due to more efficient cooling through the substrate: at the ends of the wire there is substrate to three sides rather than two as in the middle parts of the wire.

The importance of the substrate in cooling the nanowire can be seen if we use equation (4) to compute the temperature after $20 \mathrm{~ns}$ for a wire with the same geometry but without the notch and without the substrate: the heating rate is $\mathrm{d} T / \mathrm{d} t=j^{2} /(\rho C \sigma)=66.83 \mathrm{~K} / \mathrm{ns}$ as in Sec. IIIA because the geometry does not enter that calculation. Without the substrate, we would have a temperature increase of $\approx 1336 \mathrm{~K}$ after 20 nano seconds (even without taking the extra heating from the notch constriction into account).

In Fig. 3(d), the solid line shows the maximum temperature in the nanowire, the dotted (dashed) line shows 
the maximum (minimum) value of the temperature in the silicon nitride membrane substrate and the dash-dotted line shows data computed using the model by You et al 11 as a function of time over which the current pulse is applied. Note that the You model has not been derived to be used for such a thin substrate and that the large deviation for large values of $t$ is thus expected. We discuss this in detail in Sec. IV]

We can use the maximum wire temperature graph to determine the length of the current pulse that can be maintained until the temperature is pushed up to the Curie temperature, or to the material's evaporation temperature (note that the plot shows the temperature increase since the start of the experiment, not absolute temperature). If the experiment is carried out at room temperature $(\approx 300 \mathrm{~K})$, then the Curie temperature $(\approx 840 \mathrm{~K})$ is attained with a pulse duration of approximately $60 \mathrm{~ns}$. The material starts to melt and evaporate for $t \gtrsim 300 \mathrm{~ns}$, once the melting temperature of $\approx 1450^{\circ} \mathrm{C}$ is exceeded.

Asymptotically, the maximum temperature in the wire is proportional to the logarithm of pulse duration for a $2 \mathrm{~d}$ substrate and a point-like heating source. This regime is entered when the nanowire and the constriction have been heated up to a steady state, and from there on the temperature in the nanowire increases logarithmically with time while the heat front propagates from the center of the substrate disk towards the disk's boundary.

The maximum temperature in the substrate (dotted line) is assumed at the interface between the nanowire and the substrate, in the location where the nanowire is hottest. Because of the assumption of perfect thermal contact between wire and the membrane substrate, the temperature at the top of the membrane is the same as the temperature at the bottom of the wire. The difference between the maximum wire temperature and maximum substrate temperature thus provides an indication for the temperature gradient found in the wire. In Fig. 3d the two lines nearly coincide.

The dashed line in Fig. 3(d) shows the minimum value of the temperature taken across the combined system of nanowire and substrate. It starts to deviate from zero when the heat front has propagated from the center of the silicon nitride substrate disk to the boundary. In our example, that happens after $\approx 10^{4} \mu$ s when the simulated temperature increase of the wire would be over $14,700 \mathrm{~K}$. The asymptotic logarithmic behaviour of the temperature increase in the wire is maintained only until the heat front reaches the boundary of the substrate.

\section{Case study 4: Nanowire with a notch on a silicon wafer substrate}

A possible way to increase the maximum pulse length is to use a silicon wafer instead of the silicon nitride membrane, which is typically done in high frequency transport experiments. Silicon is a better conductor of heat than silicon nitride. Furthermore, the extra thickness of the wafer gives more space for heat to dissipate. In this section, we model the same nanowire with a notch geometry as above in case study 3 (Sec. IIIC) but place the nanowire on a silicon half-sphere of radius $0.5 \mathrm{~mm}$ instead of a $100 \mathrm{~nm}$ thin silicon nitride disk membrane.

Fig. 4(c) shows the temperature profile of the nanowire with notch placed on a silicon wafer after a $20 \mathrm{~ns}$ pulse (current density at the wire ends is $10^{12} \mathrm{~A} / \mathrm{m}^{2}$ ). The maximum temperature increase is $17 \mathrm{~K}$ and should be compared with Fig. 3(c) where the nanowire was placed on a (much thinner) silicon nitride substrate and the maximum temperature increase was $290 \mathrm{~K}$.

Clearly, compared to the (2d) silicon nitride membrane, the $(3 \mathrm{~d})$ silicon wafer is much more efficient at diffusing heat: the temperature increase is approximately a factor 17 smaller.

Fig. 4(d) shows the minimum and maximum temperature for the silicon wafer substrate, and the maximum temperature of the nanowire, analog to Fig. 3(d) for the nanowire on the silicon nitride membrane. We can identify three regimes: (i) for small $t$ the maximum temperature in the wire increases approximately proportional to the logarithm of time. (ii) For $1 \mu \mathrm{s} \lesssim t \lesssim 10,000 \mu \mathrm{s}$, the maximum temperature stays constant in the wire. This is the (3d) steady-state regime where the heat front propagates from the center of the silicon substrate half-sphere to the surface of the sphere. (iii) Approximately for $t \gtrsim 10,000 \mu \mathrm{s}$, the maximum wire and substrate temperatures and the minimum substrate temperature start to increase again simultaneously. This indicates that the heat front has reached the surface of the half-sphere shaped wafer substrate, and that the heat from the nanowire cannot be carried away through the propagating heat front anymore.

The maximum temperature increase of $\approx 19 \mathrm{~K}$ is reached after $\sim 1 \mu \mathrm{s}$ and is maintained until $t=10 \mathrm{~ms}$, when the heat front reaches the boundary of the wafer and the whole wafer begins to heat up. The temperature gradient from top to bottom of the wire is of the order of $5 \mathrm{~K}$ (difference of maximum wire and maximum substrate temperature).

For a system with an infinite $3 \mathrm{~d}$ substrate we expect in general that the nanowire temperature stays constant once regime (ii) of the heat front propagation has been reached. Regime (iii) would not exist for an infinite substrate, or a substrate that is efficiently cooled at its boundaries.

\section{E. Case study 5: Zigzag-shaped nanowire on a silicon wafer substrate}

In this section we investigate a nanowire geometry and experimental set up as reported in Ref. 3 where current densities of $2.2 \times 10^{12} \mathrm{~A} / \mathrm{m}^{2}$ are applied for $10 \mu \mathrm{s}$.

Figure 5(b) shows the permalloy nanowire consisting of three straight $20 \mu \mathrm{m}$ segments connected by $45^{\circ}$ bends 


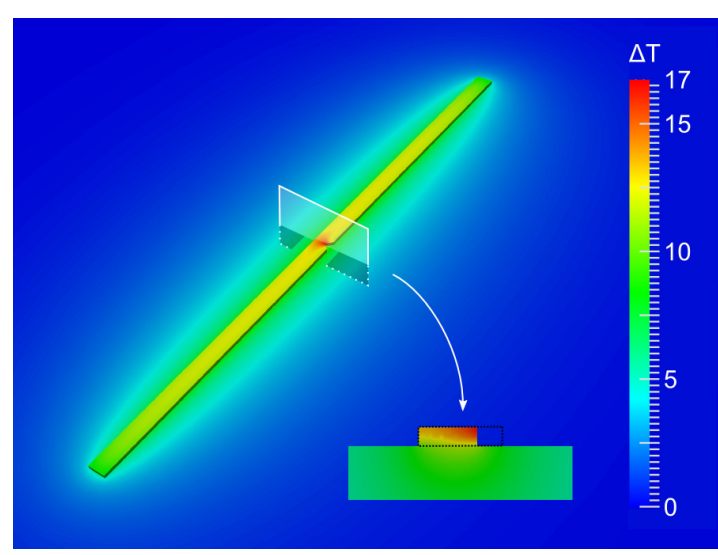

(a)

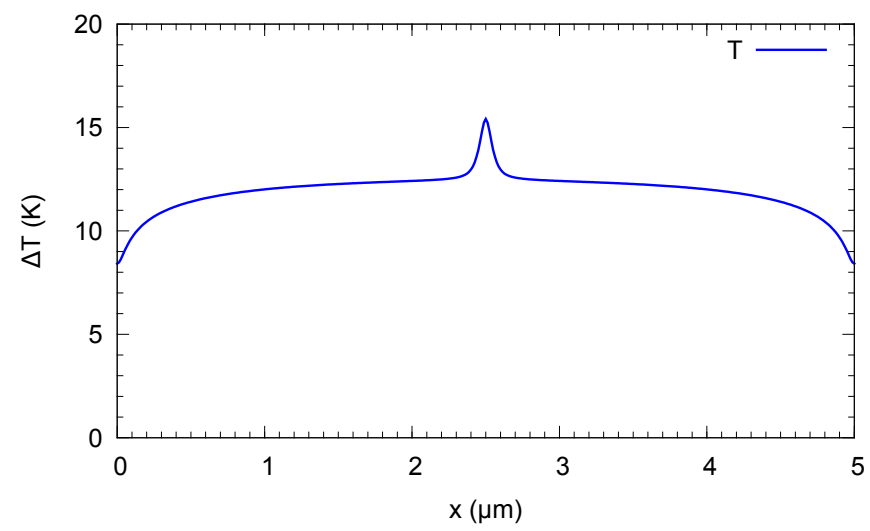

(c)

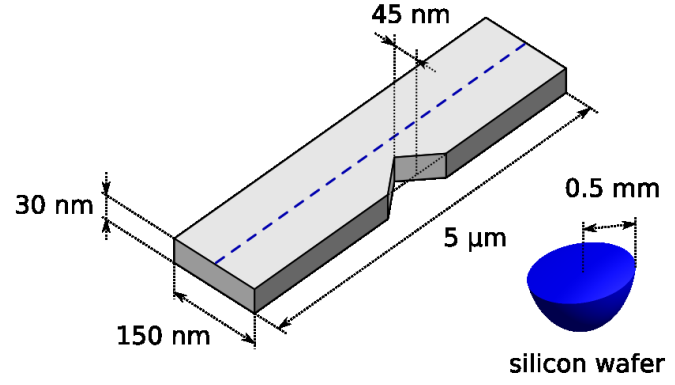

(b)

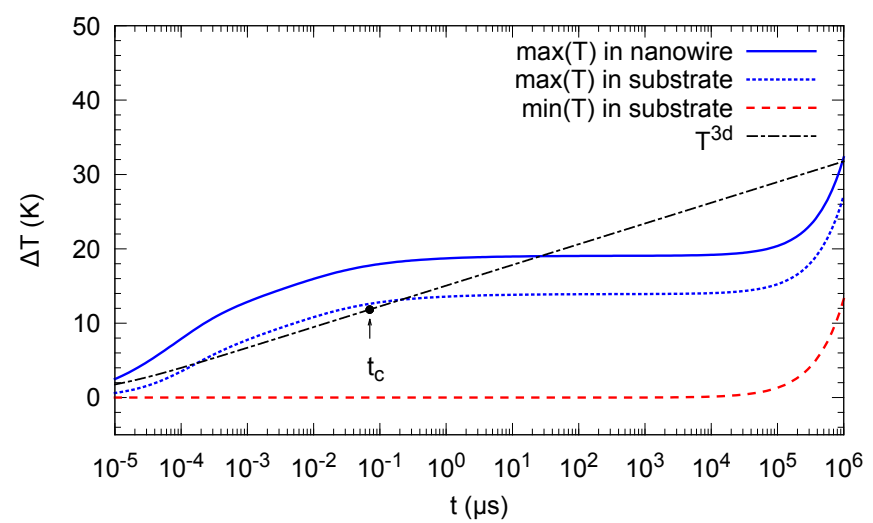

(d)

FIG. 4. Joule heating in a permalloy nanowire with a notch on a silicon wafer as discussed in Sec. IIID) (geometry of wire from Refs. 14 and 15): (a) temperature distribution $\Delta T(\mathbf{r})$ in the nanowire and substrate after $t=20$ ns, (b) geometry of the model (not to scale) and the plotting path (dashed line) used in Fig. 4(c), (c) temperature profile $\Delta T(x)$ after $t=20 \mathrm{~ns}$ along the length of the wire on a silicon wafer substrate, (d) maximum and minimum temsperatures of the silicon substrate, maximum temperature of the wire, and the prediction of the You model $T^{3 \mathrm{~d}}$ as a function of pulse length; $t_{c}$ is the characteristic time as described in Sec. IV A The silicon wafer is modelled as a half sphere (flat side attached to nanowire with notch) with radius $0.5 \mathrm{~mm}$. The current density is $j=10^{12} \mathrm{~A} / \mathrm{m}^{2}$ at the end of the wire. For material parameters see Tab. I

of radius $2 \mu \mathrm{m}$ (see also Fig. 1 in Ref. 3 ). The wire is $500 \mathrm{~nm}$ wide and $10 \mathrm{~nm}$ thick, and is placed on a silicon wafer substrate which is modelled as a silicon halfsphere of radius $0.5 \mathrm{~mm}$ as in the previous example in Sec. IIID For the simulation we scale the resistivity so that the total resistance of the device matches the value $5 \mathrm{k} \Omega$ reported in Ref. 3. This value is achieved with resistivity $\sigma^{-1}=42 \mu \Omega \mathrm{cm}$, which agrees well with published data on permalloy resistivity (Fig. 1(b) in Ref. 34, $t=10 \mathrm{~nm})$.

Figure 5(a) and 5(c) shows the temperature profile after $10 \mu \mathrm{s}$. The maximum temperature increase does not exceed $133 \mathrm{~K}$ despite the large current density and the long pulse duration. The ends of the zig-zag wire are significantly cooler (below $80 \mathrm{~K}$ ) than the center which can be attributed to more silicon wafer substrate accessible to carry away the heat that emerges from the nanowire.

Figure 5(d) shows how the maximum wire temperature (which is located in the middle segment of the zig-zag wire) and substrate minimum and maximum temperatures change over time. As before, we can identify three regimes: (i) initial heating of wire and substrate, (ii) steady state with heat front propagating through substrate and (iii) general heating of wire and substrate when heat front reaches the substrate boundary in the simulation model. Due to the larger nanowire geometry, region (ii) is less pronounced here than in Fig. 4(d)

\section{F. Case study 6: Straight nanowire on diamond substrate}

Recently, realizations of very high current densities have been reported when the permalloy nanowire is placed on a diamond substrate ${ }^{16}$ instead of silicon nitride or silicon. This finding is useful for time-integrating experiments where current excitations exceeding the microsecond timescale are required ${ }^{42 / 43}$ In this section, we 


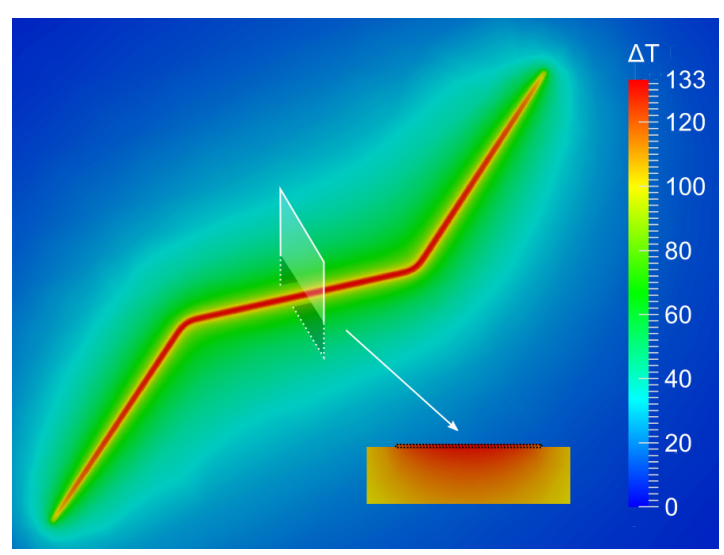

(a)

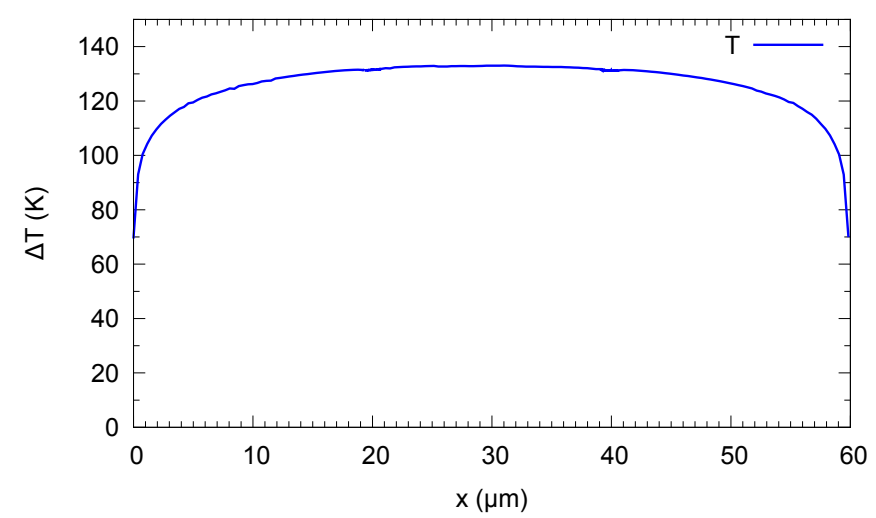

(c)

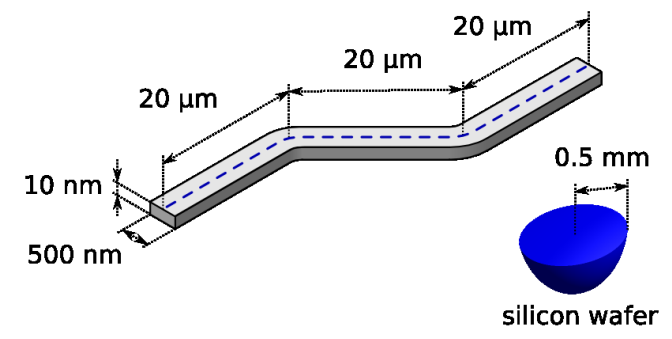

(b)

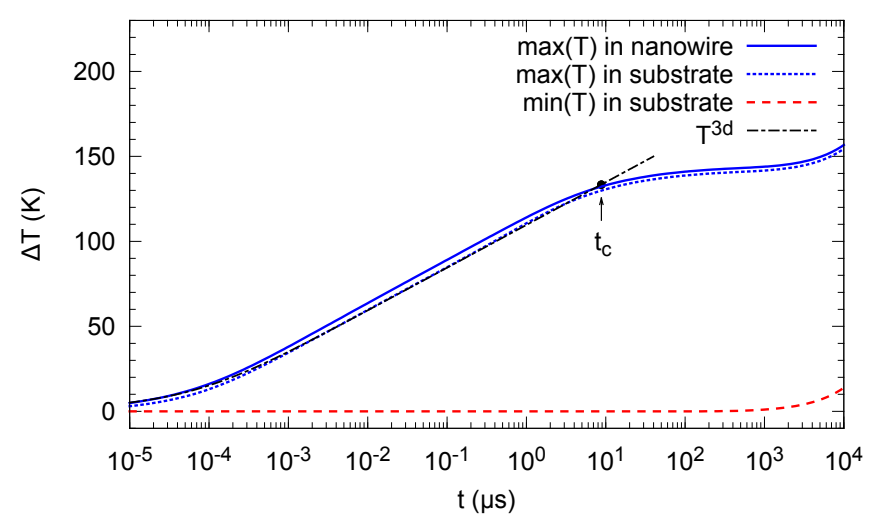

(d)

FIG. 5. Joule heating in zigzag permalloy nanowire on a silicon wafer ${ }^{3}$ (Sec. III E): (a) temperature distribution $\Delta T(\mathbf{r})$ in the nanowire and substrate after $t=10 \mu \mathrm{s}$, (b) geometry of the model (following Ref. 3) and the plotting path (dashed) for Fig. 5(c) (c) temperature profile $\Delta T(x)$ after $t=10 \mu \mathrm{s}$ along the length of the wire (following the plotting path shown in Fig. 5(b) , (d) maximum and minimum temperatures of the silicon substrate, maximum temperature of the wire, and the prediction of the You model as a function of pulse length; $t_{c}$ is calculated based on the distance between the opposite ends of the wire $L=56 \mathrm{~nm}$. The current density is $j=2.2 \times 10^{12} \mathrm{~A} / \mathrm{m}^{2}$; the resistivity of permalloy $\sigma^{-1}=42 \mu \Omega \mathrm{cm}$. For other material parameters see Tab. I]

investigate the geometry and experiment described in Ref. [16,

Figure 6(b) shows a rectangular permalloy nanowire with dimensions $25 \mu \mathrm{m} \times 650 \mathrm{~nm} \times 22.5 \mathrm{~nm}$ which is grown on a diamond crystal substrate. The thermal conductivity of diamond depends on the purity of the crystal. For the simulation, we conservatively assume a relatively impure synthetic type Ib crystal with thermal conductivity $k=1400 \mathrm{~W} /(\mathrm{K} \mathrm{m})$ (Fig. 3 in Ref. 30). A purer crystal would have higher thermal conductivity and would be more efficient at cooling the nanowire. The density and heat capacity of diamond are given in Tab. I. As before, perfect thermal contact between the wire and the substrate is assumed. In the original experiment, $\frac{16}{16}$ the whole device was placed in a cryo bath. The area of contact between the diamond crystal substrate and the bath is large compared to the size of the nanowire, therefore the temperature difference at the contact layer is likely to be small. In the simulation, the effect of the bath can be represented by using an infinite medium of diamond. In line with the case studies 4 and 5 in the previous sections, we use a half-sphere shape for the diamond substrate (radius of $0.5 \mathrm{~mm}$ ). The resistivity of the wire material is scaled to $39 \mu \Omega \mathrm{cm}$ so that the total resistance of the wire is $675 \Omega$ as in Ref. 16.

Figure 6(a) and 6(c) show the temperature profile after application of a current density of $1.5 \times 10^{12} \mathrm{~A} / \mathrm{m}^{2}$ for $1 \mu \mathrm{s}$, and Fig. 6(d) shows the maximum and minimum temperatures in the system as a function of time.

From Fig. 6(d) we can see the three regimes as in case study 4 and 5: regime (i) shows logarithmic increase of temperature with time up to approximately $0.2 \mu \mathrm{s}$. For larger $t$ the temperature remains constant during regime (ii). For $t$ greater than approximately $1000 \mu \mathrm{s}$, we reach regime (iii) where the heatfront reaches the substrate boundary. The maximum temperature increase in the wire is not exceeding $21 \mathrm{~K}$ for times smaller than $1000 \mu \mathrm{s}$, and the substrate temperature increase stays be- 


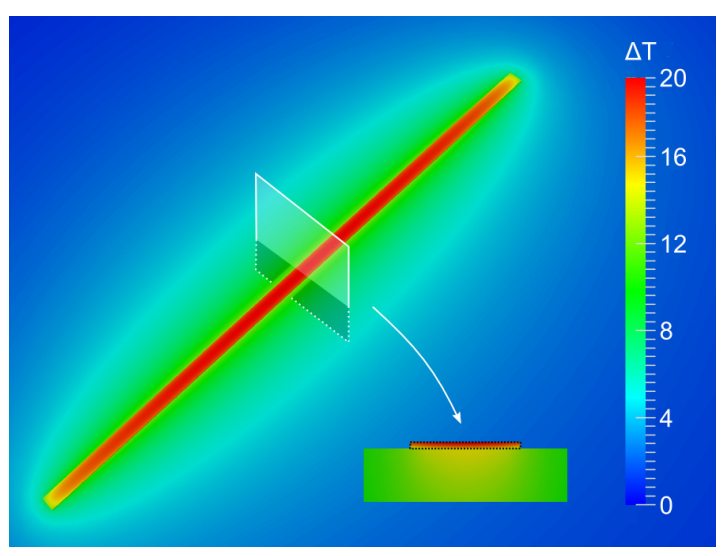

(a)

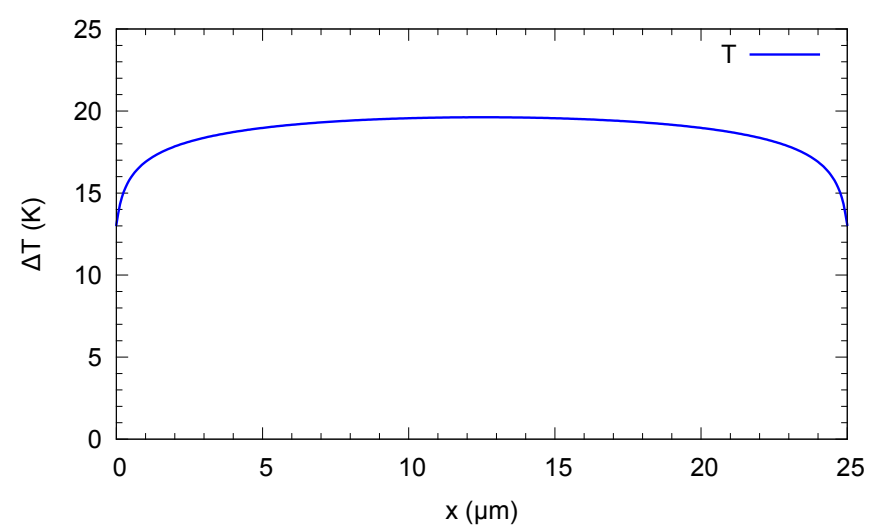

(c)

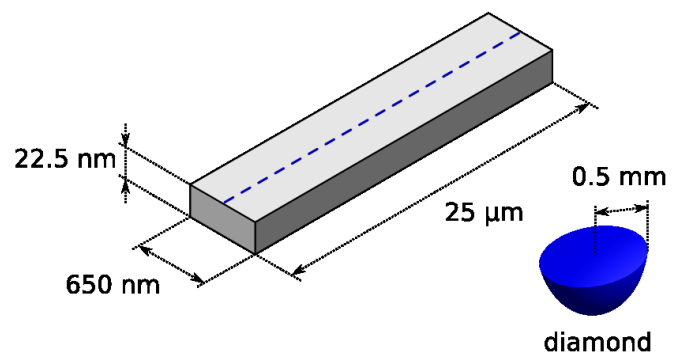

(b)

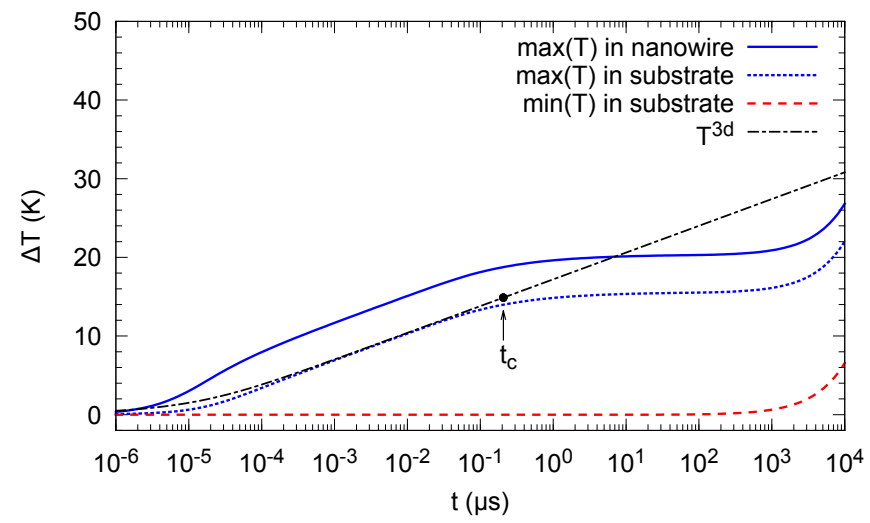

(d)

FIG. 6. Joule heating in a permalloy nanowire on a diamond crystal substrate ${ }^{16}$ (Sec. III F): (a) temperature distribution in the nanowire and substrate $T$ after $t=1 \mu \mathrm{s}$, (b) geometry of the model and the plotting path, (c) temperature profile $\Delta T(x)$ after $t=1 \mu \mathrm{s}$ along the plotting path, (d) maximum and minimum temperatures of the diamond substrate, maximum temperature of the wire, and the prediction of the You model as a function of pulse length. The current density is $j=1.5 \times 10^{12} \mathrm{~A} / \mathrm{m}^{2}$; the resistivity of permalloy $\sigma^{-1}=39 \mu \Omega \mathrm{cm}$; for other material parameters see Tab. I

low $\approx 16 \mathrm{~K}$ in that period. The plot shows that the precise current density pulse duration is not so critical: if $t$ is between $1 \mu \mathrm{s}$ and $1000 \mu$ s the nanowire attains approximately the same temperature. It is this steady state temperature increase value that should be compared with the experiment.

The difference between the maximum substrate temperature and the maximum wire temperature reflects a temperature gradient from top to bottom in the nanowire. This is just about visible in the inset in Fig. 6(a)

For larger times than $1000 \mu \mathrm{s}$, we find that the maximum and minimum temperature of the substrate and the maximum wire temperature start to increase simultaneously. This is an effect of the finite size of the substrate in the model, or, equivalently, the lack of the modelling of heat transfer away from the diamond substrate through the cryo bath. There are other, in comparison to the cryo bath less important cooling contributions, such as electric contacts, substrate holder, and surrounding gas, that have not been considered here. For the interpretation of the simulation results for the experiment in Ref. 16, we need to ignore the regime for $t \gtrsim 1000 \mu \mathrm{s}$.

The shape of the temperature profile (Fig. 6(c)] is very similar to the zigzag wire temperature profile (Fig. $5(\mathrm{c})]$.

In the original experiment, a continuous current corresponding to a current density of $1.5 \times 10^{12} \mathrm{~A} / \mathrm{m}^{2}$ heated the wire by about $230 \mathrm{~K}$ (Fig. 4 in Ref. 16).

In our simulation the nanowire changed temperature by less than $21 \mathrm{~K}$. A possible explanation for this discrepancy is that the contact and heat transfer between the wire and the diamond was imperfect. 


\section{ANALYTICAL MODELS}

\section{A. Model by You, Sung, and Joe for a nanowire on} a (3d) substrate

You, Sung, and Joe ${ }^{11}$ have provided an analytic expression $T^{3 \mathrm{~d}}(t)$ to approximate the temperature $T(t)$ of the current-heated nanowire on a three-dimensional substrate as a function of time $t$ (Eq. (16) in Ref. 11):

$$
T^{3 \mathrm{~d}}(t)=\frac{w h j^{2}}{\pi k \sigma} \operatorname{arcsinh}\left(\frac{2 \sqrt{t k /(\rho C)}}{\alpha w}\right)
$$

where $w$ and $h$ are the width and height of the wire, $\sigma$ is the wire conductivity, $j$ is the current density, and $k, \rho$, and $C$ are the thermal conductivity of the substrate, mass density of the substrate, and specific heat capacity of the substrate. We have used their adjustable parameter $\alpha=0.5$ for calculations shown in Figs. 3 to 7 .

We use name $T^{3 \mathrm{~d}}(t)$ for the equation from You, Sung and Joe - which is applicable to 3 d-substrates - to emphasize the difference to the similar looking equation for $T^{2 \mathrm{~d}}(t)$ that is derived in Sec. IV B and which is applicable to 2d-substrates.

For derivation of Eq. (5), it is assumed that the nanowire is infinitely long, and attached to a semi-infinite substrate. While the thickness $h$ and width $w$ of the wire enter the derivation to compute the Joule heating due to a given current density, the model does not allow for a temperature variation within the nanowire nor does the nanowire have a heat capacity in the model. Within this model, a heat front of (half-)cylindrical shape (cylinder axis aligned with the wire) will propagate within the substrate when the wire is heated. Thus, there is translational invariance along the direction of the wire.

We start our discussion with the zigzag nanowire as shown in Fig. 5. Figure 5(d) shows the temperature prediction of the You model as a dash-dotted line. It follows the maximum temperature in the substrate very closely for times up to approximately $2 \mu \mathrm{s}$.

At short times $t$ below 1 ns we can see the You model slightly overestimating the temperature in the nanowire in Fig. 5(d) As the model does not allow for a finite heat capacity of the wire, this is expected. As the heat capacity of the nanowire is insignificant in comparison to the substrate, this overestimation disappears if sufficient heat has been pumped into the system.

The difference between the maximum temperature in the wire and the maximum temperature in the substrate comes from a temperature gradient within the wire: the maximum temperature in the wire is at the top of the wire (which is furthest away from the cooling substrate) and the maximum temperature of the substrate is found at the top of the substrate just where the wire reaches its maximum temperature. Due to the assumption of perfect thermal contact, the bottom of the wire is exactly at the same temperature as the top of the substrate within the model description.
Since the You model does not allow for a temperature gradient within the wire, we expect its temperature prediction to follow the maximum temperature increase in the substrate. This is visible in Fig. $5(\mathrm{~d})$ for $t \gtrsim 2 \mathrm{~ns}$.

Regarding the deviation between the You model and the simulation results for $t \gtrsim 2 \mu \mathrm{s}$, we need to establish whether the required assumptions for the model are fulfilled. The You model is derived for an infinitely long wire on an infinite substrate, whereas the segments of the zigzag wire studied here have finite length. In the initial stage of heating, the temperature front in the substrate will move away from the wire sections with heat fronts aligned parallel with the wire. The heat front forms a half-cylinder (for each zigzag segment) whose axis is aligned with the wire. As long as the diameter of this half cylinder is small relative to the segment length, the wire appears locally to be infinitely long and the heat front propagates in a direction perpendicular to the wire. This is the regime where the You model is applicable, and which we have labeled as "regime (i)" in the discussion of case studies 4 (Sec. IIID) to 6 (Sec. IIIF). When the heat front has propagated sufficiently far from the nanowire to change its shape from a cylinder surface to a spherical surface, the You model is not applicable anymore. This happens approximately after $t \gtrsim 2 \mu \mathrm{s}$. We have referred to the spherical heat front propagation in the discussion above as "regime (ii)".

For the zigzag wire study the agreement of the model by You et al ${ }^{11}$ with the simulation is thus very good within the time range where the model is applicable. We note that the wire is relatively long ( $20 \mu \mathrm{m}$ per segment) and has no constriction. The You model cannot be applied for $t \gtrsim 10 \mu$ s because the finite wire length becomes important.

For the nanowire without a constriction on the diamond substrate as studied in Sec. IIIF and shown in Fig. 6, the agreement is similarly good; the You model temperature follows the substrate temperature very accurately up to $t \approx 0.1 \mu \mathrm{s}$. For larger $t$, the model becomes inaccurate as the finite length of the wire becomes important at that point.

Figure $4(\mathrm{~d})$ shows for the nanowire with a notch on the silicon substrate that for $t \gtrsim 0.1 \mu \mathrm{s}$ the gradients of both maximum temperature curves approach zero which indicates the onset of regime (ii) and implies that the You model cannot be applied for $t \gtrsim 0.1 \mu \mathrm{s}$. For smaller $t \lesssim 0.1 \mu \mathrm{s}$, the You model temperature roughly follows the maximum substrate temperature with a maximum absolute deviation of less than $3 \mathrm{~K}$.

We have carried out additional simulations (data not shown) which have demonstrated that the maximum substrate temperature line (dotted line in Fig. 4(d) is shifted down by a few degree Kelvin if the notch is removed from the geometry. The You model temperature and the maximum substrate temperature curves then coincide for $2 \lesssim t \lesssim 10 \mathrm{~ns}$. If, furthermore, we increase the wire length from $5 \mu \mathrm{m}$ to $30 \mu \mathrm{m}$, the two curves coincide 
for $2 \lesssim t \lesssim 300 \mathrm{~ns}$

Both the notch and the relative shortness of the wire decrease the accuracy of the prediction of the You model: the notch roughly shifts all temperature curves up by a few degrees whereas the length of the wire determines the time when regime (ii) is entered.

In contrast to the previous examples the nanowire with a notch in Fig. 3 is attached to a relatively thin silicon nitride membrane of thickness $100 \mathrm{~nm}$ (the silicon and diamond substrates for the discussion above are of the size order of $500 \mu \mathrm{m}$ ). The You model should not be used in the regime of membranes as the model expects an infinite substrate.

However, one could argue that the You model should be applicable for very small $t$ until the heat front emerging from the wire has propagated through the $100 \mathrm{~nm}$ thick substrate. Additional simulations (data not shown) reveal that this is the case after $t \approx 0.1 \mathrm{~ns}$.

We summarize that the You model cannot be expected to provide accurate temperature predictions for thin substrates. The deviation of the You curve in Fig. 3(d) originates in the inappropriate application of the model to a system with a thin, effectively two-dimensional, substrate.

For a 'thick', effectively three-dimensional, substrate we find that the applicability of the model is limited by the finite length of the wire. The maximum time $t_{c}$ up to which the You model is appropriate, can be estimated by calculating the characteristic time scale of the heat conduction equation (1). For a wire of length $L$, this characteristic time $t_{c}$ is

$$
t_{c} \sim\left(\frac{L}{2}\right)^{2} \frac{\rho C}{k}
$$

where $k, \rho$, and $C$ are the thermal conductivity, density and specific heat capacity of the substrate material. The greater the nanowire length, the longer it takes for the heat front to assume spherical shape around the nanowire heating source. The greater the heat capacity $(\rho C)$ and the smaller the thermal conductivity, the slower is the propagation of the heat front in the substrate. The current density does not enter the equation as it only affects the temperature and not the time or length scale.

Substituting the corresponding parameters for each case study, we compute the characteristic time $t_{c}$ for the nanowire with a notch in case study 4 (Sec. IIID) to be $70 \mathrm{~ns}$, for the zigzag nanowire in case study 5 (Sec. IIIE) to be $8.8 \mu \mathrm{s}$, and for the nanowire on diamond in case study 6 (Sec. IIIF) to be $0.2 \mu \mathrm{s}$. We have added these values to the figures Fig. $4(\mathrm{~d}), 5(\mathrm{~d})$, and $6(\mathrm{~d})$ and they are in good agreement with the corresponding finite element results.

The time $t_{c}$ marks the transition from regime (i) to regime (ii).

In summary, we find that the You model provides an accurate description of the maximum substrate temper- ature if used within its bounds of applicability, i.e. during regime (i) for three-dimensional substrates. In the You model, the nanowire has no heat capacity and this results in the model slightly overestimating the temperature for very small $t$ (visible for example in Fig. $5(\mathrm{~d})$ for $t \lesssim 1 \mathrm{~ns})$. The temperature within the nanowire can show a gradient (hotter at the top, cooler at the interface to the substrate), and the You model computes the smaller temperature in the wire. For the studies carried out here we find this temperature difference to be less than $10 \mathrm{~K}$ in all cases although this difference depends on material parameters and wire thickness. The You model cannot be applied for thin, effectively two-dimensional, substrates such as the membrane substrate case study in Sec. IIIC and Fig. 3 .

\section{B. Analytic expression for nanowire on a membrane ( $2 \mathrm{~d}$ substrate)}

Equation (5) is valid for pulse durations up to the critical duration $t_{\mathrm{c}}$ (Eq. (6) ) for effectively three-dimensional substrates, i.e. substrates whose thickness is sufficiently large so that the heat front in the substrate does not reach the substrate boundary for $t<t_{\mathrm{c}}$. This condition is fulfilled for the case studies in Sec. IIID, IIIE, and IIIF. If the substrate is effectively two-dimensional (such as in Sec. III C), then Eq. (5) cannot be applied. In this section, we show how Eq. (5) can be adapted to the $2 \mathrm{~d}$ case.

The assumptions made in the derivation of Eq. (5) require a system of nanowire and substrate that is translationally invariant in one direction. In Sec. IIID IIIF this direction was along the long axis of the wire, and we refer to this axis as $x$, and assume that the height $h$ of the wire extends along the $z$ axis.

For the case of a nanowire on a thin membrane substrate, we can regard the system as two-dimensional by assuming invariance in the perpendicular direction. In other words, to apply a modified version of Eq. (5), we assume that the temperature distribution in the membrane system is invariant along the $z$ axis. We model the nanowire as embedded in the substrate (not grown on top of the substrate as in the real system) and imagine an increase of thickness of both the wire and the substrate such that they expand from $-\infty$ to $+\infty$ in z-direction. The cooling and heating in each slice (in the $x-y$ plane) of the nanowire and substrate system is not affected by cooling and heating from slices above and below; it does not matter whether we consider only one isolated slice (as in the real system) or imagine an infinite stack of slices closely packed on top of each other.

In more detail, we first convert the system of the nanowire of height $h$ and substrate of thickness $d$ to a $2 \mathrm{~d}$ system of equal height. We increase the height of the nanowire by a factor $c=d / h$ so that the wire and the substrate are now both of height $d$ (assuming that generally $d>h$ but the derivation also holds for $d<h$ ). This 
increases the volume of the wire by a factor of $c$, and thus we will have to correct down the heat emerging from the wire by the same factor at a later point.

Second, to obtain translational invariance in the $z$ direction we imagine a stack of such identical $2 \mathrm{~d}$ systems on top of each other. Using the substitutions $w \rightarrow L$ and $h \rightarrow w$, we obtain

$$
\begin{aligned}
T^{2 \mathrm{~d}}(t) & =\frac{L w j^{2}}{\pi k \sigma} \frac{1}{c} \frac{1}{2} \operatorname{arcsinh}\left(\frac{2 \sqrt{t k /(\rho C)}}{0.5 L}\right) \\
& =\frac{w h L j^{2}}{2 d \pi k \sigma} \operatorname{arcsinh}\left(\frac{2 \sqrt{t k /(\rho C)}}{0.5 L}\right)
\end{aligned}
$$

The first fraction in Eq. (7) is based on Eq. (5) and includes the substitutions $w \rightarrow L$ and $h \rightarrow w$, and we also substitute $w \rightarrow L$ in the denominator of the arcsinh argument, and use $\alpha=0.5$. The second fraction $(1 / c)$ reduces the temperature increase by $c$ to compensate for the increase of heating by the factor $c$ above when we increased the thickness of the nanowire to the thickness of the substrate. The third fraction (1/2) in Eq. (7) is a correction because the nanowire is now surrounded by substrate in all directions, and not only in one half-space as in Eq. (5), thus the cooling is twice as efficient and the temperature increase is halved.

Equation (8) can be used to compute the maximum temperature increase $T^{2 \mathrm{~d}}(t)$ for a nanowire of length $L$, width $w$, and height $h$ on a two-dimensional substrate of thickness $d$.

In contrast to $T^{3 \mathrm{~d}}(t)$ there is no upper time limit $t_{\mathrm{c}}$ for the validity of Eq. (8) as the emerging heat-front will always stay translationally invariant.

The comparison of $T^{2 \mathrm{~d}}(t)$ with the finite element simulation results from case study 3 (Sec. III C) is shown in Fig. 7. The overall agreement with the simulation results is good for all times $t$. The heat capacity of the wire is not considered in the model for $T^{2 \mathrm{~d}}(t)$ which is reflected in the overestimation of the temperature in Fig. 7. From comparative simulations with different material parameters, we find that the agreement of the two curves is better for reduced heat capacity of the wire, and better for increased thermal conductivity of the substrate. The effect of the finite heat capacity of the wire becomes less important for longer current pulses, and the two curves in Fig. 6 become closer for larger $t$ (not shown here).

\section{PERPENDICULAR NANOWIRES}

Recent progress in sample growth has allowed to create free-standing nanowires which are grown perpendicular to their substrate (for example 44 46). While it is outside the scope of this work to investigate these systems in detail, we comment briefly on possible analytical approximations. For such a free standing nanowire, the analytical expression (4) is a good first approximation to compute its temperature increase as a function of applied current density duration.

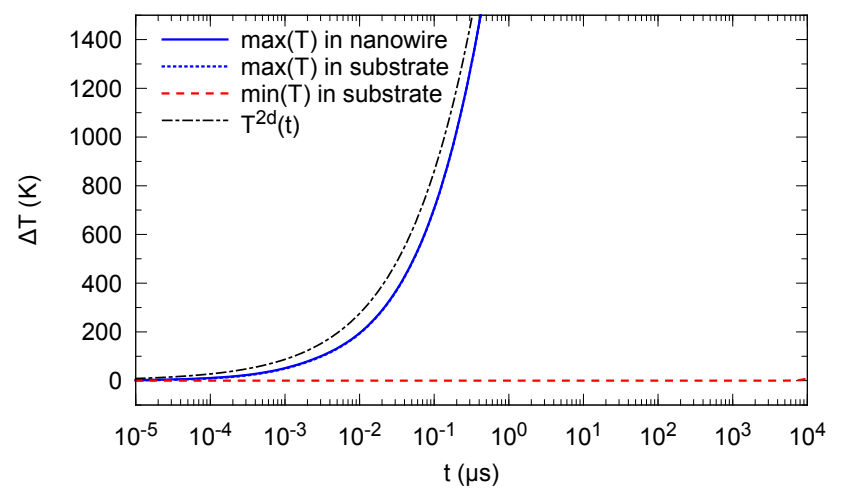

FIG. 7. Comparison of simulated wire temperature from case study 3 (Sec. III C) with substrate temperatures obtained using the analytical expression $T^{2 \mathrm{~d}}(t)$ from Eq. (8), as a function of pulse length.

For the temperature increase $T_{\perp}^{3 \mathrm{~d}}(t)$ of a perpendicular nanowire which is completely embedded in a substrate material (such as an $\mathrm{Al}_{2} \mathrm{O}_{3}$ matrix), a variation of equation (5) can be employed:

$$
T_{\perp}^{3 \mathrm{~d}}(t)=\frac{1}{2} T^{3 \mathrm{~d}}(t)
$$

In contrast to the nanowire mounted on top of a halfspace filling substrate (5) as studied in section III the substrate is here space-filling, and thus twice as effective in cooling the system.

\section{SUMMARY}

We have carried out detailed numerical simulations of the current distribution, Joule heating, and dissipation of temperature and heat through the nanowire and the substrate for a number of experiments and three different substrate types. We find that the silicon nitride membrane (thickness $100 \mathrm{~nm}$ ) is the least efficient in cooling a nanowire that experiences Joule heating. Due to the quasi-two-dimensional nature of the membrane, the temperature in the nanowire will keep increasing proportionally to the logarithm of time for longer current pulses while the heat front (forming a circle in the membrane substrate) propagates away from the nanowire, which is located in the center of the heat front circle.

Using a (effectively three-dimensional) silicon wafer substrate instead of the (effectively two-dimensional) silicon nitride membrane, there is a qualitative change: once the steady state is entered, the heat front propagates in three dimensions and keeps the temperature in the heated nanowire constant. In addition to the better cooling through the three-dimensionality of a silicon wafer, the cooling is improved further by the thermal conductivity of silicon which is more than one order of magnitude greater than that of silicon nitride (see Tab. I).

If we replace silicon in the $3 \mathrm{~d}$ substrate with diamond, the cooling is improved again significantly: diamond's 
thermal conductivity is about an order of magnitude greater than that of silicon (see Tab. I).

In addition to these generic insights, we have worked out the temperature increase quantitatively for a series of recent experimental publications. ${ }^{3 / 13}-16$ The model simulations presented here show for all of them that the temperature increase due to the Joule heating did not result in the temperature exceeding the Curie temperature.

We compare these results to the approximating but analytical model expression provided by You, Sung, and Joe ${ }^{11}$ and investigate the limits of its applicability. We provide an estimate for the characteristic time $t_{c}$ over which the You model is valid for three-dimensional substrates.

Finally, we provide a new analytical expression that allows to compute the temperature for a nanowire on a two-dimensional substrate in the presence of Joule heating. This expression should be of significant value in the design and realization of spin-torque transfer studies on membranes where experimenters are often operating very close to the Curie temperature or even the melting temperature, and where no estimate of the wire's temperature has been possible so far.

We provide supplementary online materia $\sqrt{47}$ to compute $T^{2 \mathrm{~d}}(t)$, and $T^{3 \mathrm{~d}}(t)$ for other materials and experimental parameters such as current density and current pulse duration.

The research leading to these results has received funding from the European Community's Seventh Framework Programme (FP7/2007-2013) under Grant Agreement n233552 (DYNAMAG), and from the EPSRC (EP/E040063/1 and Doctoral Training Centre Grant EP/G03690X/1). Financial support by the Deutsche Forschungsgemeinschaft via SFB 668 as well as GK 1286 and from the Cluster of Excellence "Nanospintronics" funded by the Forschungs- und Wissenschaftsstiftung Hamburg is gratefully acknowledged. We are also grateful for the support from ANSYS Inc.

We thank Sebastian Hankemeier for useful discussions, and the anonymous reviewers for their constructive and helpful feedback.

* fangohr@soton.ac.uk

1 T. Kamionka, M. Martens, K. W. Chou, M. Curcic, A. Drews, G. Schütz, T. Tyliszczak, H. Stoll, B. Van Waeyenberge, and G. Meier, Phys. Rev. Lett., 105, 137204 (2010)

2 A. Yamaguchi, T. Ono, S. Nasu, K. Miyake, K. Mibu, and T. Shinjo, Phys. Rev. Lett., 92, 077205 (2004).

3 M. Kläui, P.-O. Jubert, R. Allenspach, A. Bischof, J. A. C. Bland, G. Faini, U. Rüdiger, C. A. F. Vaz, L. Vila, and C. Vouille, Phys. Rev. Lett., 95, 026601 (2005).

4 G. Meier, M. Bolte, R. Eiselt, B. Krüger, D.-H. Kim, and P. Fischer, Phys. Rev. Lett., 98, 187202 (2007)

5 S. Lepadatu, M. C. Hickey, A. Potenza, H. Marchetto, T. R. Charlton, S. Langridge, S. S. Dhesi, and C. H. Marrows, Phys. Rev. B, 79, 094402 (2009)

${ }^{6}$ S. Parkin, M. Hayashi, and L. Thomas, Science, 320, 190 (2008)

S. S. P. Parkin, Tech. Rep. (2004) u.S. Patent No. 3096 834005.

8 S. Urazhdin, N. O. Birge, W. P. Pratt, and J. Bass, Phys. Rev. Lett., 91, 146803 (2003).

${ }^{y}$ M. Laufenberg, W. Bührer, D. Bedau, P.-E. Melchy, M. Kläui, L. Vila, G. Faini, C. A. F. Vaz, J. A. C. Bland, and U. Rüdiger, Phys. Rev. Lett., 97, 046602 (2006).

10 F. Junginger, M. Kläui, D. Backes, U. Rüdiger, T. Kasama, R. E. Dunin-Borkowski, L. J. Heyderman, C. A. F. Vaz, and J. A. C. Bland, Appl. Phys. Lett., 90, 132506 (2007)

11 C. You, I. Sung, and B. Joe, Appl. Phys. Lett., 89, 222513 (2006)

12 P. Bruno, Phys. Rev. Lett., 83, 2425 (1999)

13 M.-Y. Im, L. Bocklage, P. Fischer, and G. Meier, Phys. Rev. Lett., 102, 147204 (2009)

${ }^{14}$ L. Bocklage, B. Krüger, T. Matsuyama, M. Bolte, U. Merkt, D. Pfannkuche, and G. Meier, Phys. Rev. Lett., 103, 197204 (2009)

is H. H. Langner, L. Bocklage, B. Krüger, T. Matsuyama, and G. Meier, Applied Physics Letters, 97, 242503 (2010).

16 S. Hankemeier, K. Sachse, Y. Stark, R. Frömter, and H. Oepen, Appl. Phys. Lett., 92, 242503 (2008).

17 Ansys Inc, "Ansys, version 12.0," (2010), http://www.ansys.com.

18 Multiphysics Modeling and Engineering Simulation Software, "Comsol multi-physics version 4.0," (2008), http://www.comsol.com/.

19 T. Fischbacher and H. Fangohr, ArXiv e-prints, 0907.1587 (2009), http://arxiv.org/abs/0907.1587, arXiv:0907.1587 [physics.comp-ph].

20 T. Fischbacher, M. Franchin, G. Bordignon, and H. Fangohr, IEEE Transactions on Magnetics, 43, 2896 (2007), http://nmag.soton.ac.uk.

21 G. Meier, personal communication (2010).

22 D. Bonnenberg, K. A. Hempel, and H. P. J. Wijn, "SpringerMaterials - The Landolt-Börnstein Database," (Springer) Chap. 1.2.1.2.10 Thermomagnetic properties, thermal expansion coefficient, specific heat, Debye temperature, thermal conductivity, p. 252.

23 C. Y. Ho, M. W. Ackerman, K. Y. Wu, S. G. Oh, and T. N. Havill, J. Phys. Chemical. Ref. Data, 7, 959 (1978).

24 E. A. Owen, E. L. Yates, and A. H. Sully, Proceedings of the Physical Society, 49, 315 (1937)

20 C. H. Mastrangelo, Y.-C. Tai, and R. S. Muller, Sensors and Actuators A: Physical, 23, 856 (1990), ISSN 09244247.

26 E. Domalski and E. Hearing, "NIST Chemistry WebBook, NIST Standard Reference Database Number 69," (NIST) Chap. Condensed Phase Heat Capacity Data.

27 C. Y. Ho, R. W. Powell, and P. Liley, Thermal Conductivity of the Elements: A Comprehensive Review (AIP, 1978).

28 H. Bettin, M. Glaser, F. Spieweck, H. Toth, A. Sacconi, A. Peuto, K. Fujii, M. Tanaka, and Y. Nezu, IEEE Transactions on Instrumentation and Measurement, 46, 556 (1997), ISSN 0018-9456.

${ }^{29}$ E. Domalski and E. Hearing, "NIST Chemistry WebBook, NIST Standard Reference Database Number 69," (NIST) 
Chap. Condensed Phase Heat Capacity Data.

${ }^{30}$ Y. Yamamoto, T. Imai, K. Tanabe, T. Tsuno, Y. Kumazawa, and N. Fujimori, Diamond and Related Materials, 6, 1057 (1997), ISSN 0925-9635.

${ }_{31}$ P. Ownby and R. Stewart, "Engineered materials handbook," (ASM International, 1991) Chap. Engineering Properties of Diamond and Graphite, pp. 821-834.

${ }^{32}$ H. Fangohr, J. P. Zimmermann, R. P. Boardman, D. C. Gonzalez, and C. H. de Groot, J. Appl. Phys., 103, 07D926 (2008)

33 Y. Wang, C. H. de Groot, D. Claudio-Gonzalez, and H. Fangohr, Applied Physics Letters, 97, 262501 (2010)

${ }^{34}$ L. K. Bogart and D. Atkinson, Appl. Phys. Lett., 94, 042511 (2009).

35 Silson Ltd., Northampton, United Kingdom. http://www.silson.com.

36 X. Zhang and C. P. Grigoropoulos, Review of Scientific Instruments, 66, 1115 (1995), ISSN 0034-6748.

37 A. Irace and P. M. Sarro, Sensors and Actuators A: Physical, 76, 323 (1999), ISSN 0924-4247.

38 B. L. Zink and F. Hellman, Solid State Communications, 129, 199 (2004), ISSN 0038-1098.

${ }^{39}$ R. Sultan, A. D. Avery, G. Stiehl, and B. L. Zink, Journal of Applied Physics, 105, 043501 (2009), ISSN 0021-8979.
40 L. Thomas, M. Hayashi, X. Jiang, C. Rettner, and S. S. P. Parkin, Appl. Phys. Lett., 92, 112504 (2008)

41 J. Crangle and G. C. Hallam, Proceedings of the Royal Society of London. Series A, Mathematical and Physical Sciences, 272, 119 (1963).

${ }^{42}$ L. Heyne, J. Rhensius, D. Ilgaz, A. Bisig, U. Rüdiger, M. Kläui, L. Joly, F. Nolting, L. J. Heyderman, J. U. Thiele, and F. Kronast, Phys. Rev. Lett., 105, 187203 (2010)

45 B. Krüger, M. Najafi, S. Bohlens, R. Frömter, D. P. F. Möller, and D. Pfannkuche, Phys. Rev. Lett., 104, 077201 (2010)

${ }^{44}$ K. Nielsch, F. Müller, A. Li, and U. Gösele, Advanced Materials, 12, 582 (2000), ISSN 0935-9648.

${ }^{45}$ L. Vila, P. Vincent, L. Dauginet-De Pra, G. Pirio, E. Minoux, L. Gangloff, S. Demoustier-Champagne, N. Sarazin, E. Ferain, R. Legras, L. Piraux, and P. Legagneux, Nano Letters, 4, 521 (2004).

${ }^{46}$ K. Pitzschel, J. Bachmann, S. Martens, J. M. MonteroMoreno, J. Kimling, G. Meier, J. Escrig, K. Nielsch, and D. Görlitz, Journal of Applied Physics, 109, 033907 (2011).

47 MS Excel file to compute $T^{2 \mathrm{a}}(t)$ and $T^{\mathrm{sa}}(t)$ [URL will be inserted by AIP]. 